\title{
Watching Ancient Paintings through Synchrotron-Based X-Ray Microscopes
}

\section{Cotte and J. Susini}

Even if attitudes are changing, our study of works of art using synchrotron instruments is usually perceived to be exotic. To the community of curators and conservators, synchrotron instruments can appear as enormous, and sometimes frightening, exotic machines. Likewise, for the community of chemists and physicists working with synchrotron sources, works of art can be seen as complex and exotic samples. However, these two communities are finding an increasing interest in collaborating. On the one hand, scientific analyses aim to complement historical knowledge and assist curators in the future preservation of artworks. On the other hand, museum objects will certainly become more and more appealing in materials research as they can provide researchers with examples of very longterm evolutions, hardly reproducible at the laboratory scale.

Paintings, and more generally all cultural heritage objects, have inestimable value, not only from artistic, aesthetic, and historic points of view, but also because they give us a unique testimony to the technical, physical, and chemical practices through the ages. Some materials, such as natural pigments, may intrinsically retain information about their geographical origins, and can be used to identify the trade routes of the ancient world. In parallel, artificial materials used in arts and crafts provide evidence of the evolution of ancient technologies. Accessing the chemical formulation of paintings can be particularly rewarding, providing us with new insight into techniques and knowledge developed by the artists and artisans years, decades, or even centuries ago.

Long-term changes in the chemical composition of materials encompass another important area where detailed analysis is required. The understanding of degradation processes is essential to enable curators and conservators to identify the best techniques to use to preserve a given object.

From the chemistry perspective, paintings can be seen as the archetype of puzzling materials. They often present a complex multilayered structure, with layer sizes ranging from a half millimeter to some micrometers. These layers can be non-uniform, containing pigment grains of a few micrometers.

As already mentioned, these objects are particularly precious. Hence, non-invasive techniques are usually favored for analysis. When we are allowed to acquire a sample, in particular for a deeper analysis of the entire stratigraphy, the fragment size is generally only a few hundred micrometers. After embedding the fragment in resin, we polish it perpendicularly to the painting surface, to reveal the whole multilayered structure (see Figure 1c). Various analyses can then be carried out, with different nondestructive microanalytical techniques.

\section{From the chemistry perspective, paintings can be seen as the archetype of puzzling materials.}

Paintings are a complex mixture of tens of ingredients: inorganic and crystallized products, such as pigments and plasters; organic and amorphous ingredients, such as binders, sizers, or varnishes; and hybrid materials which can result from the reaction between the two former classes of compounds. Getting a complete identification of these complex compositions often implies the use of complementary analytical techniques.

These ingredients may evolve with time, chemically and physically. Some evolutions, such as oil drying, can be intentional, while others, such as pigment discoloration, can be fortuitous. In some cases-for example, the metallic carboxylates formed by the reaction of metallic ingredients with organic binders-the distinction between controlled, shortterm reactions, and accidental mid- or long-term reactions, is not straightforward. This multi-speed kinetic is a daily problem in our research activities.

Classically, chemical observations are made with optical and electron microscopes. More recently, synchrotron radiationbased microscopes have emerged as promising instruments for the study of paintings, and more generally of cultural heritage objects. The photon beam generated by electrons accelerated inside the synchrotron ring has unique properties that are particularly well-suited for the analysis of ancient paintings.

For example, the high source brightness (i.e., intensity per unit source size and divergence) enables working with micrometric and even submicrometric probes,

INTERFACES seeks articles featuring the people and profession of materials. Send proposals to Bulletin@mrs.org. with reduced dwell time, without compromising data quality. The combination of these three aspects is fundamental for chemical imaging: the chemical analysis can be performed, step by step, on a twodimensional array, and sometimes on a three-dimensional volume. Such methods make possible the observation of the entire painting stratigraphy.

Also, a wide range of chemical information is accessible, from elemental (thanks to micro x-ray fluorescence) to molecular (thanks to Fourier transform infrared microscopy, and micro x-ray absorption spectroscopy), to structural (with micro x-ray diffraction). The microanalytical platform provided by synchrotron facilities such as the European Radiation Synchrotron Facility (ESRF), Grenoble, allows us to characterize most of the ingredients present in paintings, and more generally, most of the materials constituting museum objects.

Characterizing degradation processes can be particularly tricky, since the averaged elemental composition may remain the same in both original and altered regions. In this context, methods probing the chemical environment of specific atoms, such as micro x-ray absorption near-edge spectroscopy ( $\mu$ XANES), can be very rewarding. This technique can give, for example, information about the oxidation states of an element, or about its neighboring atoms. Metal corrosion is a good candidate for such applications: We can use XANES to evaluate the oxidation state of the metal and to identify alteration products, in particular at the surface of the metallic objects. This technique has the unique potential to provide the means to do selective analysis of one element of interest, even if the analyzed compounds are amorphous and diluted in very complex mixtures.

The following example illustrates the potential of synchrotron-based $\mu$ XRF and $\mu$ XANES. It deals with a frequent but incompletely understood degradation phenomenon occurring in paintings: cinnabar blackening. Cinnabar, a red mercury sulfide (HgS) pigment, has been known and employed from antiquity. Many Pompeian paintings present a flamboyant red background made with this vivid pigment. Unfortunately, this pigment is not always stable. It can turn to white, grey, or black, which dramatically damages the aspect of the paintings, all the more so as this pigment is usually extensively employed to paint backgrounds or dresses. In 2005, we conducted our first 

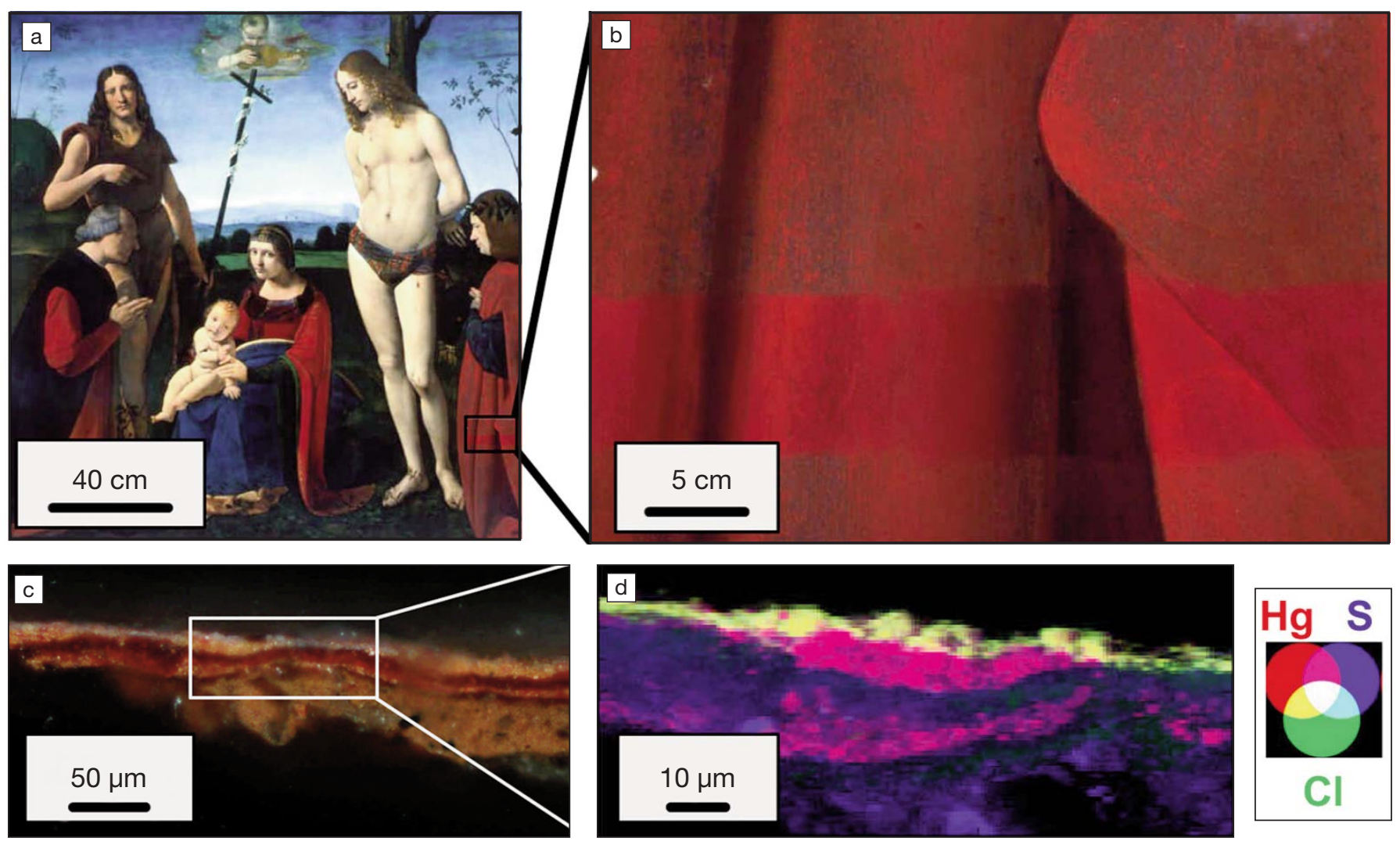

Figure 1. (a) Madonna with Child, St. Sebastian, St. John the Baptist and two donors, Boltraffio, Louvre (@ C2RMF); (b) zoom on the donor dress with blackening cinnabar partially cleaned in 1995 (OC2RMF); (c) a transversal cross-section of a painting fragment, showing a succession of two cinnabar layers, and a very thin grey film, on top; (d) elemental analysis by synchrotron-based micro x-ray fluorescence (ID21, ESRF), with red-green-blue visualization. Mercury is plotted in red, sulfur in blue, and chlorine in green. Yellow regions correspond to mixtures of mercury and chlorine, while pink regions correspond to mixtures of mercury and sulfur.

analysis on Pompeian paintings. These paintings showed an intense red background when they were discovered, but a few years after the excavation, they exhibited a sad grey-black aspect.

Metacinnabar, which is a black mercury sulfide formed from cinnabar by action of light, was originally hypothesized. Then $\mu \mathrm{XRF}$ and $\mu$ XANES analyses were performed at the ESRF. The x-ray microscope at the ID21 beamline is set up specifically for elemental and chemical identification, at the micrometer scale, and with high sensitivity. A wide range of samples (from biological, environmental, medical, chemical, and physical sciences) pass through this instrument on a weekly basis.

In the case of Pompeian paintings, the $\mathrm{x}$-ray analyses did not reveal metacinnabar, but rather two other chemical families: some black layers of gypsum (calcium sulfate), and some mercury sulfide/chloride compounds. ${ }^{*}$ Two potential origins

*M. Cotte, J. Susini, N. Metrich, A. Moscato, C. Gratziu, A. Bertagnini, and M. Pagano, Anal. Chem. 78 (21) (2006), p. 7484. for chloride compounds were advanced: the vicinity to the Mediterranean salt water sea, or the possible use of a particular wax used to cover some paintings and described by ancient naturalists such as Pliny the Elder as being made of wax mixed with sea water.

Two years later, in 2007, we continued this research with the study of Gothic Spanish paintings. The Monastery Pedralbes, Barcelona, founded in 1326 by Queen Elisenda de Montcada, was under restoration. The purpose of the cleaning action, directed by J. Chillida, was to remove a posterior white lime wash applied onto Gothic paintings. Behind a more or less thick layer of gypsum, the curators found the original pigments. Some flower features were made with red pigment, but a black film was observed inbetween this red layer and the lime wash coating. In order to decide if the black layer had to be removed during the cleaning, some chemical analyses were performed. Very small fragments were collected, at various situations, where degradation was occurring (in the cloister, exposed to the exterior, in particular to sun light), but also in nondegraded regions (inside the Church). As in the Pompeian case, mercury chloride compounds were observed. More precisely, it was possible to image the progression of the alteration. The red original cinnabar layer was covered with a first grey mercury chloride-sulfide compound-corderoite $\mathrm{Hg}_{3} \mathrm{~S}_{2} \mathrm{Cl}_{2}$ type; second, by a grey mercury-chloride compoundcalomel $(\mathrm{HgCl})_{2}$ type; and, finally, by the ulterior gypsum- $-\mathrm{CaSO}_{4} \cdot 2 \mathrm{H}_{2} \mathrm{O}$ - constitutive of the lime wash. Thanks to the synchrotron-based x-ray microscope, the different steps of cinnabar "digestion" by chloride could be observed in a single analysis. With a unique chemical snapshot, a sort of complete biography of the paintings could be obtained. As in the case of Pompeian paintings, the proximity to the sea was hypothesized as the main source of chlorine. It was confirmed also that light contributes to the process. It is probable that the blackening happened a long time ago, and it may have motivated the ulterior (unfortunately unreferenced) application of lime wash, aiming at hiding 
these decomposing paintings. ${ }^{+}$As it would have been difficult to prevent any further contamination and consequent reaction of chlorides on the re-discovered Gothic paintings, Chillida decided to restrict his action to the unoriginal lime wash, and to leave the homogeneous grey mercury-chloride layer at the painting surface, which indeed may act as a protective coating to ulterior inner alterations.

Last year, the story continued, from wall paintings to canvas and panels. We wanted to see if similar alteration processes could be observed on paintings preserved in museum conditions, in particular in the Louvre, Paris, which is not particularly close to the sea. The analysis was carried out on several paintings, among which was the Madonna with Child, St. Sebastian, St. John the Baptist and two donors, painted by Giovanni Antonio Boltraffio in 1500. In this painting, cinnabar alteration is particularly visible on the dress of the donor, on the right side (facing painting) (Figure 1a). In 1995, restoration was undertaken, in particular to clean this dress. Two rectangular cleaning tests were performed and are still visible today (Figure 1b). A few painting fragments were also sampled to carry out chemical analyses.

From observations of the painting surface, the degradation seems rather important. Surprisingly, it is in fact difficult to detect the grey layer on transversal crosssections (Figure 1c). Visible and chemical observations show that the alteration layer is often less than $10 \mu \mathrm{m}$. The sensitivity of the laboratory techniques available in 1995 was insufficient to characterize the grey film. Since then, the embedded fragments were kept in the "samples base" of the Center of Research and Restoration of French Museums (C2RMF, Paris), waiting for the development of more precise and adapted analytical instruments.

In December 2008, these fragments traveled from Paris to the European Synchrotron Radiation Facility, in Grenoble, to be analyzed by micro x-ray techniques. $X$-ray fluorescence analyses revealed that the very thin superficial degradation layer contained traces of chlorides. The $\mu$ XANES enabled the identification of some mercury chlorides, confirming that chlorides had reacted with the original pigment. This example is particularly interesting because, for some reason, two cinnabar layers were superimposed, separated by a red lacquer. One can clearly observe that chlorine is limited to a very superficial layer, and is not present inside the painting (neither in

tM. Cotte, J. Susini, V.A. Sole, Y. Taniguchi, J. Chillida, E. Checroun, and P. Walter, J. Anal. At. Spectrom. 23 (2008), p. 820. the exterior nor in the interior cinnabar layer). Consequently, an intrinsic origin of the chloride can be refuted. Contrarily, we can assume that chlorides came from some superficial application, or environmental contamination. In the present case, one possible source of chlorine could be the carbon tetrachloride used in 1986 to treat the panel against woodworms. Such a chemical is no longer used; curators prefer the anoxia treatment, with which chlorine contamination is avoided.

This work is going on in the frame of a larger project, combining the analysis of ancient paintings with the reconstruction and artificial aging of cinnabar paintings, as well as with thermodynamic simulations to better estimate the environmental effects (e.g., light, humidity, and chloride) on cinnabar blackening. This step will be necessary to establish adapted strategies for restoration and long-term conservation of works of art.

Beyond the cultural heritage field, such analyses can also find impact in the environmental science domain, as cinnabar mobility in nature can induce mercury pollution, and subsequently potential toxicity.

$M$. Cotte is a research scientist at the Center of Research and Restoration of the French Museums at the Centre National de la Recherche Scientifique in Paris. She is also a visiting scientist at the European Synchrotron Radiation Facility.

J. Susini is the head of the Instrumentation Services and Development Division (ISDD) at the European Synchrotron Radiation Facility in Grenoble.

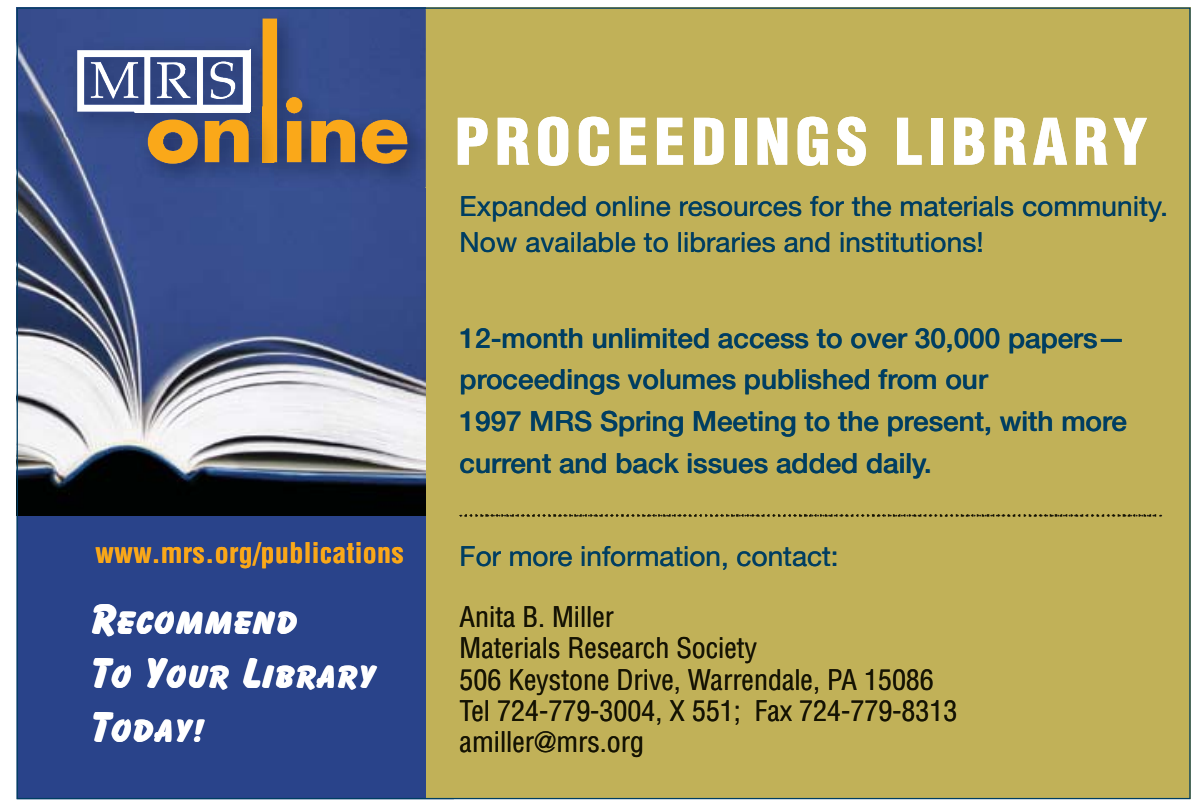

Advertisers in This Issue

Page No.

Advanced Metallization Conference (AMC) 2009 431

American Chemical Society, Neutron Reflectivity

High Voltage Engineering

Outside back cover

Janis Research Company 414

National Electrostatics Corp. 454

J.A. Woollam Co, Inc. 440

For free information about the products and services offered in this issue, check http://www.mrs.org/bulletin ads. 